\title{
Analgesics and its Adverse Effects
}

\section{Rajat Kr Sachdeva*}

Dean, Director at Dr. Sachdeva's Dental Institute, Delhi, India

*Corresponding Author: Rajat Kr Sachdeva, Dean, Director at Dr. Sachdeva's Dental Institute, Delhi, India.

Received: June 03, 2019; Published: August 21, 2019

Analgesics also referred to as "PAIN KILLERS", relieve the sub ject from pain without hampering its consciousness. According $t_{1}$ their time of onset and duration of action they can either be shor acting painkiller or long acting pain killer.

Although NSAIDs (non-steroidal anti- inflammatory drugs) anc Opioids are frequently used, but there widespread use has causeu significant health issues.

The injudicious use of analgesics

- $\quad$ Pain killers when consumed in more than normal dosage can lead to certain unpleasant side effects like nausea, vomiting and diarrhea, as the digestive tract is comprisei of opioid receptors that interacts with the drugs.

- Analgesics when administered intra-venously can cause long-term heart damage and other cardiovascular problems.

- $\quad$ Another health hazard that may occur is the risk of con tracting blood-borne diseases if analgesics are injectei through non-sterile or re-used needles.

What will happen if analgesic is consumed more than the prescribed dosage?

It can prove to be fatal. Amongst analgesics, especially Opioids is amongst the common drug consumed intentionally as user have a sensation of euphoria when the drug first hits their brains. This is the reason why reports to its addiction have been reported commonly. This in turn will affect the threshold value of analgesics to act and you'll require large dose of the particular drug to achieve the same result - and that puts you at risk of an overdose.

Reactions of body in case of overdosage

- $\quad$ During an overdose, the drug affects those parts of brain that are responsible for circulatory and respiratory functions.
- $\quad$ The pulse rate drops, breathing becomes slow and heart rate decreases. In some people dysarrythmia can occur, that may lead to cardiac arrest.

- $\quad$ The respiratory and circulatory function ceases. The amount of oxygen reaching various organs diminishes, and ultimately the brain may get affected. It can lead to seizures and irreversible brain damage.

- The signs of an overdose of painkillers includes:

- Cold, clammy skin due to excessive sweating

- Blue or grey lips and nails

- Slow or absent breathing

- Gurgling sounds

- Confusion/Disorientation

- $\quad$ Vomiting

- $\quad$ Tiny pupils

- Unresponsiveness

- Constipation/Itchiness

Analgesic effects on pregnancy

Pregnancy care is one of the greatest challenges in medicine as it demands a special care in the use of drugs because of altered physiology. About 15-20 percent of birth defects are attributed to occur due to certain maternal drug exposures.

- $\quad$ Paracetamol: Low birth weight and pre-term deliveries have been observed with chronic usage.

- Salicylates: Cases of birth defects, decreased weight during birth, neonatal hemorrhage and prolonged labor have been reported with this drug.

- Indomethacin: It may result in premature closure of the fetal ductus arteriosus, fetal nephrotoxicity and pulmonary hypertension of the newborn. 
Pain killers and alcohol

Both pain killers and Alcohol depresses the Cental nervous system. Consuming both together can result in a more severe depressive effect. Its combined use can develop

- Brain damage

- Liver damage

- $\quad$ Damage to CVS/GIT

Plus, there is an increased potential to develop addiction/physical dependence.

Analgesics and their adverse effects

- $\quad$ Opiates

As opiates have a wide range of side effects.

It is frequently abused drug. The reason for their abuse is that they interact at various opioid receptors in the brain, preventing a chemical known as GABA from being released which is responsible for the release of dopamine, which causes its tremendous increased level creating the feeling of euphoria.

Adverse effects may include

- $\quad$ Constipation and itching which may require long-term treatment.

- $\quad$ Decreased central respiratory drive, respiratory rate which may lead to ineffective ventilation.

- $\quad$ Fatalities have been reported in patients with obstructive sleep apnoea who are prescribed opioids.

- $\quad$ Paracetamol: The most common type of painkiller.

Adverse effects of paracetamol

Its overdosage is linked with liver damage and kidney damage. Alcohol should be avoided or if not the consumed in very less amount along with paracetamol.

- Aspirin (acetylsalicylic acid): Used for moderate pain conditions.

\section{Adverse effects of aspirin}

If you possess following characteristics, then you have to consult doctor for taking pain killers apart from aspirin.

- $\quad$ Pregnancy Lactating mothers

- liver or kidney diseases

- asthmatic patients

- $\quad$ Peptic ulcers

- $\quad$ Patients having bleeding disorder

- Have had an allergic reaction to aspirin or NSAIDs
If any of these apply to you, ask a doctor or pharmacist for advice before taking NSAIDs.

This drug is not recommended in children below 15 years.

Ibuprofen: (NSAID) for pain and inflammation and is readily available.

\section{Adverse effects of ibuprofen}

Not recommended in patients under following conditions :

- $\quad$ Pregnancy/Lactating

- $\quad$ Past allergic reaction to aspirin or NSAIDs

- $\quad$ Stomach ulcers

- Having severe heart failure or liver disease

- Asthmatic Patients

- Lupus erythematosus

- Kidney or liver problems

- $\quad$ Ever had any bleeding in your stomach

- High blood pressure/stroke

- Heart problems - such as angina, heart attacks, or mild or moderate heart failure.

Topical analgesics

- $\quad$ Topical analgesics are commonly used for minor strains and sprains and include camphor, menthol, methyl salicylate, methyl nicotinate and capsacin.

- Patients on blood thinners will have prolonged prothrombin time if used for a long span.

- Discontinue its application if you notice redness or blistering of the skin.

- $\quad$ Avoid using any heating devices in conjunction with it.

This high usage rate is combined with inadequate level of awareness about the associated health risks, which raises several concerns about patients safety.

Volume 2 Issue 9 September 2019 (C) All rights are reserved by Rajat Kr Sachdeva. 